\title{
Neuronal Cyclic AMP Controls the Developmental Loss in Ability of Axons to Regenerate
}

\author{
Dongming Cai,, ${ }^{1}$ Jin Qiu, ${ }^{1}$ Zixuan Cao, ${ }^{1}$ Marietta McAtee, ${ }^{2}$ Barbara S. Bregman, ${ }^{2}$ and Marie T. Filbin ${ }^{1}$ \\ ${ }_{1}^{1}$ Department of Biological Sciences, Hunter College, City University of New York, New York, New York 10021, and \\ 2Department of Neuroscience, Georgetown University Medical School, Washington, DC 20007
}

\begin{abstract}
Unlike neonatal axons, mammalian adult axons do not regenerate after injury. Likewise, myelin, a major factor in preventing regeneration in the adult, inhibits regeneration from older but not younger neurons. Identification of the molecular events responsible for this developmental loss of regenerative capacity is believed key to devising strategies to encourage regeneration in adults after injury. Here, we report that the endogenous levels of the cyclic nucleotide, cAMP, are dramatically higher in young neurons in which axonal growth is promoted both by myelin in general and by a specific myelin component, myelin-associated glycoprotein (MAG), than in the same types of neurons that, when older, are inhibited by myelin-MAG. Inhibiting a down-
\end{abstract}

The adult mammalian CNS does not regenerate after injury, although young axons do regrow (Bates and Stelzner, 1993; Hasan et al., 1993). Changes in the cellular environment and in the neuronal response to that environment are both responsible for the absence of regeneration by mature axons (Keirstead et al., 1992; Li and Raisman, 1993; Schwab and Bartholdi, 1996). However, adult CNS neurons have not lost the intrinsic capacity to regrow, because, when provided with a permissive environment, they will extend long processes (Richardson et al., 1980; David and Aguayo, 1981; Crutcher, 1989; Schnell and Schwab, 1990; Schwab et al., 1993; Cheng et al., 1996). Therefore, some new component in the adult CNS environment actively inhibits axonal growth after injury. One obvious candidate that appears with late development in the CNS is myelin. Indeed, many studies in vivo and in vitro have shown that myelin inhibits axonal regeneration in adult neurons (Crutcher, 1989; Schnell and Schwab, 1990; Keirstead et al., 1992; Cheng et al., 1996). Strong evidence that myelin is a major factor in preventing regeneration was presented when extensive regeneration occurred in mice that had been immunized with myelin before spinal cord transection (Huang et al., 1999). Extensive regeneration occurred because the myelin antibodies neutralized myelin-specific inhibitors of regrowth, allowing regeneration to commence immediately after injury. On

\footnotetext{
Received Jan. 16, 2001; revised March 21, 2001; accepted April 17, 2001.

This work was supported by grants from the National Multiple Sclerosis Society (NMSS), the National Institutes of Health (NIH) (NS 37060 to M.T.F. and NS19259 to B.S.B.), and a core facility grant from the Research Centers for Minorities Institute-NIH. J.Q. is a fellow of the NMSS. We thank Dr. Arthur McMorris and Dr. Anthony Weimelt for providing the cAMP antibody, Dr. Roger Persell for critically reading this manuscript, and Dr. Lloyd Williams for his assistance with the image analysis.

D.C. and J.Q. contributed equally to this study.

Correspondence should be addressed to Dr. Marie T. Filbin, Biology Department, Hunter College, 695 Park Avenue, New York, NY 10021. E-mail: filbin@genectr.hunter.cuny.edu.

Copyright (C) 2001 Society for Neuroscience $0270-6474 / 01 / 214731-09 \$ 15.00 / 0$
}

stream effector of cAMP [protein kinase $A(P K A)$ ] prevents myelin-MAG promotion from young neurons, and elevating cAMP blocks myelin-MAG inhibition of neurite outgrowth in older neurons. Importantly, developmental plasticity of spinal tract axons in neonatal rat pups in vivo is dramatically reduced by inhibition of PKA. Thus, the switch from promotion to inhibition by myelin-MAG, which marks the developmental loss of regenerative capacity, is mediated by a developmentally regulated decrease in endogenous neuronal cAMP levels.

Key words: axonal regeneration; cAMP; protein kinase $A$; myelin; MAG; development the other hand, myelin does not exert this inhibitory effect on embryonic neurons, either in culture (Shewan et al., 1995) or when transplanted in vivo (Li and Raisman, 1993). Identification of the molecular difference between embryonic and adult neurons that is responsible for this change in regenerative capacity has long been believed to be the key to encouraging adult axons to regrow after injury.

Along with a developmental change in axonal response to myelin in general, it has been reported that a number of different neurons respond to an individual myelin-specific protein, myelinassociated glycoprotein (MAG), by switching from promotion to inhibition during development (McKerracher et al., 1994; Mukhopadhyay et al., 1994; DeBellard et al., 1996). For retinal ganglion (RG) neurons and spinal neurons, the switch has occurred by birth; embryonic RG and spinal neurons are promoted by MAG, whereas postnatal axonal growth is inhibited (Salzer et al., 1990; DeBellard et al., 1996; Turnley and Bartlett, 1998). For dorsal root ganglion (DRG) neurons, the switch occurs postnatally with a sharp transition from promotion to inhibition by MAG at postnatal day 3-4 (P3-4) (Johnson et al., 1989; Mukhopadhyay et al., 1994; DeBellard et al., 1996). Although the appearance of myelin is an obvious change in the environment with development, the molecular basis for the significant developmental shift in the response of neurons to MAG and myelin is not known. However, the response of neurons to MAG in culture is paralleled by their response to myelin in general, which in turn is indicative of how they regenerate in vivo.

Recently, we showed that elevating endogenous levels of cAMP effectively blocked the inhibition of axonal regeneration by MAG and myelin (Cai et al., 1999). Consistent with this observation, others showed that not only is repulsion of growth cones by MAG dependent on cAMP, but so is the attraction of growth cones to other guidance cues (Ming et al., 1997; Song et al., 1997, 1998). Importantly, it was also shown that for these 
molecules, inhibition could be converted to attraction by elevating neuronal cAMP, and attraction can be converted to inhibition by blocking a downstream effector of cAMP, protein kinase A (PKA). The question now raised by all of these studies is whether endogenous levels of cAMP influence the spontaneous switch in response of neurons to $\mathrm{MAG}$ and myelin and the loss of regenerative capacity observed during development.

\section{MATERIALS AND METHODS}

Neurite outgrowth on cells or myelin. Rat RG neurons and DRG neurons were isolated as described previously (DeBellard et al., 1996). For raphespinal projecting neurons, the most caudal region of the raphe nucleus was removed, and the neurons were dissociated in $0.025 \%$ trypsin for $10 \mathrm{~min}$ at $37^{\circ} \mathrm{C}$. The neurite outgrowth on myelin or on transfected Chinese hamster ovary $(\mathrm{CHO})$ cells was as described (Mukhopadhyay et al., 1994; Cai et al., 1999), with the following modifications. The neurite outgrowth assay was performed by adding $5 \times 10^{4}$ neurons to the immobilized myelin substrate or $2 \times 10^{4}$ neurons to the $\mathrm{CHO}$ cell monolayers. Where indicated, db-cAMP (1 mM), db-cGMP (1 mM), H89 $(2 \mu \mathrm{M})$, KT2750 (200 nM), KT5823 $(1 \mu \mathrm{M})$, Rp-cAMP $(20 \mu \mathrm{M})$, or Sp-cAMP $(50 \mu \mathrm{M})$ was added to the cultures. All types of neuron cultures were immunostained for growth-associated protein 43 (GAP43) as described before (Mukhopadhyay et al., 1994), but for raphe nuclei neurons, because the cultures were not pure, cultures were also stained for serotonin. A goat serotonin antibody (DiaSorin, Stillwater, MN) was used at 1:1000. For double staining, GAP43 antibody was detected with anti-rabbit Oregon Green-conjugated secondary antibody, and serotonin antibody was detected with anti-goat biotinylated secondary antibody followed by Texas Red-conjugated streptavidin. Approximately $98 \%$ of the cultures were serotonin-positive. For DRG and RG neurons, the length of the longest neurite for each GAP43-positive neuron for the first 180-200 neurons encountered when scanning the slide in a systematic manner was determined using an Oncor image analysis program. The same effects were obtained when total processes, rather than the longest neurite, were measured. For raphe neurons, only those neurons that were both GAP43- and serotonin- positive were measured. Neurite measurements were compared between groups using a one-way ANOVA.

Immunoassay for cAMP. For each cAMP assay, neurons were dissociated, and cyclic AMP was measured immediately using a competitive immunoassay, according to the manufacturer's (Amersham, Arlington Heights, IL) instruction . For each assay, $1 \times 10^{6} \mathrm{RG}$ cells or $2 \times 10^{5}$ DRG or raphe nuclei neurons were plated into each of six wells. Each assay was repeated at least four times.

Immunofluorescence staining for cAMP. cAMP immunostaining was performed on DRG cryosections with a rabbit polyclonal antiserum according to Wiemelt et al. (1997). Isolated, intact DRGs were fixed with acrolein for $1 \mathrm{hr}$ at room temperature (RT), incubated in $20 \%$ sucrose until equilibrium, embedded with optimum cutting temperature (OCT) matrix (Tissue-Tek; Miles Inc., Elkhart, IN), and cryosectioned at $5 \mu \mathrm{m}$. The fixed RG neurons or DRG cryosections were washed in a quenching solution containing $1 \mathrm{mg} / \mathrm{ml}$ glycine for $15 \mathrm{~min}$ at RT, followed by $1 \%$ $(\mathrm{w} / \mathrm{v})$ sodium cyanoborohydride for $15 \mathrm{~min}$. Then the slides were stained by incubating them overnight at $4^{\circ} \mathrm{C}$ in the anti-cAMP antiserum diluted $1: 50$ in a solution containing $5 \%$ goat serum and $0.5 \%$ Triton X-100. Then, the binding of the primary antibody was visualized using the biotin-streptavidin system.

Spinal cord lesions and transplants. Pups (2-3 d of age) were anesthetized by hypothermia, and under sterile surgical conditions, a laminectomy was made at the T6 spinal cord level. An overhemisection lesion was made in all animals $(n=38)$. The following experimental groups were examined: lesion plus H-89 $(n=10)$, lesion plus saline $(n=10)$, lesion plus transplant plus H-89 $(n=11)$, and lesion plus transplant plus saline $(n=7)$. The saline or $\mathrm{H}-89(0.5 \mathrm{~mm})$ was delivered via Gelfoam placed at the lesion or lesion plus transplant site. Transplants of embryonic day 14 spinal cord were prepared as described previously (Bregman and McAtee, 1993).

Tracing and immunohistochemistry. Two weeks after lesion, the anterograde tracer, biotin dextran amine (BDA, 10,000 molecular weight; Molecular Probes, Eugene, OR), was used to study changes in the descending corticospinal tract fibers. After anesthesia (choral hydrate, $400 \mathrm{mg} / \mathrm{kg}$ ), the sensorimotor cortex was exposed, and a $10 \%$ solution of BDA in sterile saline was injected into the cortex bilaterally $(0.3-0.5 \mu \mathrm{l}$ per injection, $3 \mu$ l total volume per motor cortex). After the injections, the dura was covered with Gelfoam soaked in saline, and the overlaying skin was sutured. Two weeks after the BDA injection, the rats were anesthetized with chloral hydrate and perfused transcardially with $0.9 \%$ saline followed by ice-cold $4 \%$ paraformaldehyde in $0.1 \mathrm{M}$ phosphate buffer. Tissue was passed through graded sucrose solutions and prepared for immunohistochemistry using the modified techniques of Veenman et al. (1992) and Herzog and Brosamle (1997). Briefly, cryostat sections were washed with phosphate buffer and incubated in an avidin-biotinperoxidase complex for $90-120 \mathrm{~min}$ at room temperature (Vector Elite ABC Kit; Vector Laboratories, Burlingame, CA), and the BDA-filled axons were visualized using a solution of diaminobenzidine tetrahydrochloride-nickel ammonium sulfate and hydrogen peroxide. Adjacent sections were processed immunocytochemically for visualization of serotonergic axons (serotonin; DiaSorin) as described previously (Bregman et al., 1997; Diener and Bregman, 1998). Cellular morphology within the transplants was evaluated in sections stained with cresyl violet or neutral red. The extent of axonal growth was evaluated by individuals unaware of the experimental group to which the animals belonged. The extent of axonal growth within the transplants was scored according to a rating scale. No growth within the transplant was scored as 0; dense growth throughout the transplant equivalent to that observed in previous studies was rated as +++ . Only sections in which the transplant was contiguous with the host spinal cord and without any intervening glial or cystic barrier were scored. Sections from seven of the transplant plus H89 animals and six of the transplant plus saline-treated animals were scored.

\section{RESULTS}

\section{Endogenous cAMP levels in DRG neurons decrease with development and parallel the developmental loss of ability to grow on myelin}

Previously, we reported that MAG, like many axon guidance molecules (Colamarino and Tessier-Lavigne, 1995; Song et al., 1998) was bifunctional. When cultured on CHO cells that were transfected to express MAG, the neurite length from rat DRG neurons older than $\mathrm{P} 4$ and through to adult was, on average, 50\% shorter than those grown on control $\mathrm{CHO}$ cells that were not expressing MAG. In contrast, MAG promoted neurite outgrowth from DRG neurons from animals younger than $\mathrm{P} 4$, and the transition from promotion to inhibition by MAG occurred sharply at P3-4 (Mukhopadhyay et al., 1994; DeBellard et al., 1996). Now we show that on a substrate of purified myelin, DRG neurons follow a very similar trend in response. When cultured on myelin, DRG neurons from P1 animals extend relatively long processes (longest neurite on average, $25 \mu \mathrm{m}$ ), which then show diminished length with age (Figs. $1 A, 2 A$ ). By P5, neurite length is about fivefold shorter than neurites from P1 animals. Extension of short neurites from DRG neurons on myelin persists through to adults. On a control substrate such as poly-L-lysine or $\mathrm{CHO}$ cells not expressing MAG, there is no decrease in neurite length with age. Therefore, like the age-related switch in response to MAG, DRG neurons grown on myelin at the same age also switch from extending relatively long neurites at $\mathrm{P} 1$ to extending either very short or no neurites by P5 (Figs. $1 A, 2 A$ ). We already know that artificially elevating cAMP in older neurons blocks inhibition by not only MAG but also myelin in general (Cai et al., 1999). Therefore, it is reasonable to suggest that the better growth of young neurons on myelin-MAG is a consequence of higher endogenous levels of cAMP relative to older neurons. To determine whether this is the case, cAMP levels were measured directly in DRG neurons of different ages, immediately after their removal from the animal. In concert with the sharp transition in neurite growth response to myelin and MAG, there is a concomitant sharp drop in the endogenous levels of cAMP in DRG neurons at P3-4 to less than one-tenth the levels at birth (Fig. $1 B)$, where they persist to adult. Consistent with this measure of absolute cAMP levels of dissociated DRG neurons, when immu- 
$\mathbf{A}$

DRG Neurons on Myelin

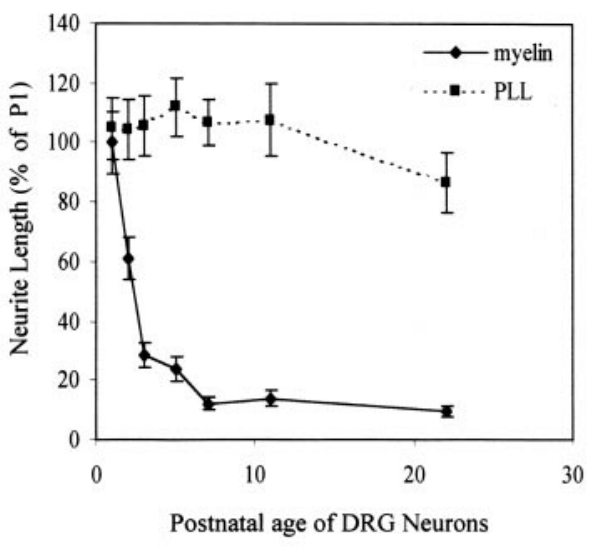

B

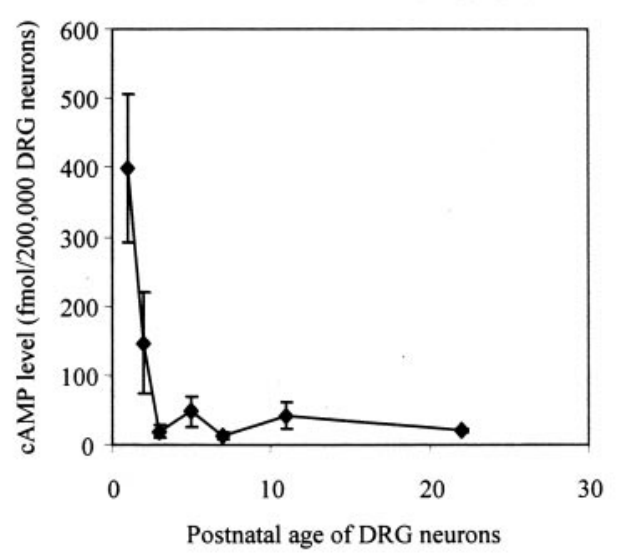

C

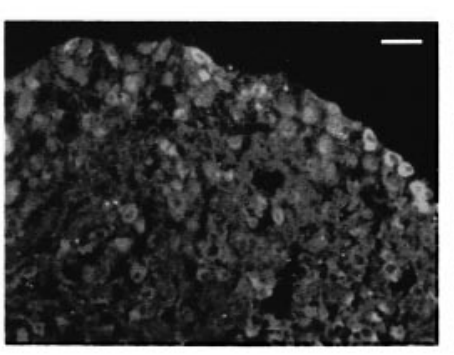

P0

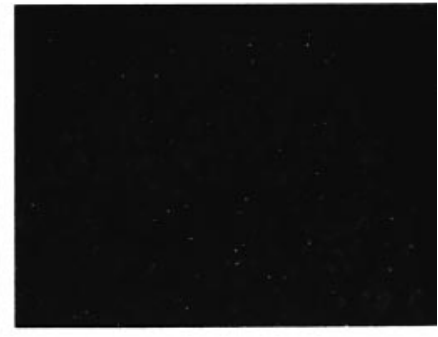

P5
Figure 1. Developmental changes in neurite outgrowth of DRG neurons on myelin correlate with changes in endogenous levels of cAMP. Dissociated DRG neurons from P1-P22 animals, as indicated, were cultured overnight on a substrate of purified rat CNS myelin or poly-L-lysine; afterward, they were fixed and immunostained for GAP43. In each experiment, at each DRG age, the mean length of the longest GAP43positive neurite for 180-200 neurons was measured ( \pm SEM) for at least three separate experiments. Results are presented as percentage of P1 neurite length $(A)$. To measure cAMP, $2 \times 10^{5}$ dissociated DRG neurons from P1-P22 animals, as indicated, were plated into each well, and cAMP was measured using a competitive immunoassay. Results are the mean $( \pm \mathrm{SE})$ of at least three experiments, each performed in sextuplicate $(B)$. DRG neurons were removed from P0 and P5 animals, fixed immediately, and immunostained for cAMP $(C)$. Scale bar, $50 \mu \mathrm{m}$. nostained in situ with a cAMP antibody (Wiemelt et al., 1997), there is obviously much more cAMP in P0 than in P5 DRG neurons (Fig. 1C).

\section{High levels of endogenous cAMP in young DRG neurons account for the promotion of neurite outgrowth by MAG and myelin}

To determine whether the high levels of cAMP in newborn DRG neurons are indeed required for promotion of neurite outgrowth by myelin and MAG, the downstream effector of cAMP, PKA, was inhibited. Both the PKA inhibitor, KT5720 (200 nM), and the cAMP antagonist, Rp-cAMP $(20 \mu \mathrm{M})$, blocked neurite outgrowth on myelin by $>50 \%$ and completely blocked promotion by MAG from P1 DRG neurons in culture; an inhibitor of protein kinase $\mathrm{G}(\mathrm{PKG})$, KT5823 (1 $\mu \mathrm{M})$, had no effect (Fig. $2 A-C)$. None of these compounds had an effect on neurite outgrowth on control cells not expressing MAG or on growth on MAG-expressing cells of DRG neurons older than P4 (data not shown). In addition, the cAMP analog, db-cAMP, had no effect on the good growth of P1 DRG neurons on MAG cells or myelin (data not shown). Together with our previous findings that artificially elevating cAMP in older DRG neurons blocks inhibition by myelin-MAG, these results strongly suggest, first, that promotion and inhibition of axonal growth by myelin-MAG are each cAMP-dependent and, second, that the endogenous levels of cAMP in DRG neurons dictate the developmental switch of these neurons to inhibition.

Changes in neurite outgrowth of retinal ganglion neurons on myelin and MAG correlate with, and are dependent on, changes in endogenous levels of cAMP

Because many other neurons switch their response to MAG and myelin with development, we now asked whether cAMP dependence is a general mechanism involved in the switch or if it is restricted to DRG neurons. Retinal ganglion neurons are also known to switch in response to MAG, but unlike DRG neurons, the switch for RG neurons occurs embryonically (Salzer et al., 1990; DeBellard et al., 1996). Because the endogenous levels of cAMP are likely to change during the culture period required (days) to isolate RG neurons from other RG cells, cAMP levels were measured for mixed populations of RG cells immediately after removal from the animals. We found that the endogenous levels of cAMP in embryonic day 18 (E18) RG cells are at least threefold higher than P5 RG cells (Fig. $3 A$ ); the cAMP levels remain consistently low in all postnatal $\mathrm{RG}$ cells up to adult (data not shown). When grown on myelin as a substrate, embryonic RG neurons (distinguished from other cell types by their morphology and by staining with a second immunological marker, Thy-1; data not shown) extended relatively long, highly branched processes after overnight culture, whereas P5 RG neurons extended neurites that were about fivefold shorter and much less complex (Fig. $3 B$ ). When either of the PKA inhibitors, KT5720 or Rp-cAMP, was included in the cultures, neurite outgrowth from E18 RG neurons on myelin was greatly reduced, whereas an inhibitor of PKG had no effect (Fig. 3B,C).

In a similar fashion, the promotion of neurite outgrowth by MAG from E18 RG neurons was blocked by the same PKA inhibitors and not by inhibitors of PKG (Fig. 4A). Furthermore, as with DRG neurons older than P5, inhibition of neurite outgrowth from postnatal RG neurons grown on myelin or MAG was blocked if either db-cAMP or Sp-cAMP, but not db-cGMP, was included in the cultures (Figs. $3 B, C, 4 B$ ). None of the compounds used had any effect on neurite outgrowth on control cells (data not shown). Together, these results suggest that cAMP 
A
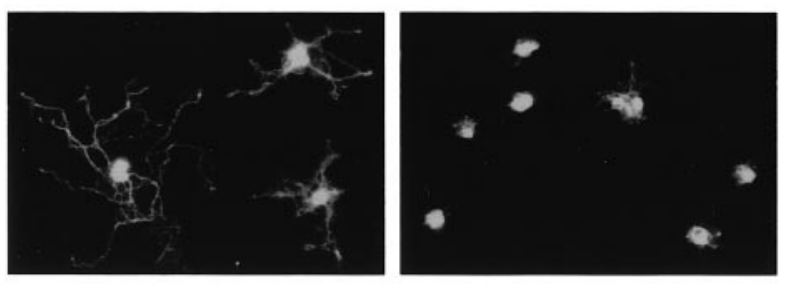

P1

Figure 2. Developmental changes in neurite outgrowth of DRG neurons on myelin and MAG are dependent on changes in endogenous levels of cAMP. Dissociated DRG neurons from P1 or P5 animals, as indicated, were cultured overnight on a substrate of purified rat CNS myelin $(A, B)$, MAG-expressing cells (striped bars), or control cells (black bar) (C). Where indicated, $200 \mathrm{nM}$ KT5720 $(+K T), 20 \mu \mathrm{M}$ Rp-cAMP $(+R p)$, or $1 \mu \mathrm{M}$ KT5823 (+PKG Inh.) was added during culture; afterward, they were fixed and immunostained for GAP43. Scale bar (shown in $A$ ), $10 \mu \mathrm{m}$. In each experiment, at each DRG age, the mean length of the longest GAP43-positive neurite for 180-200 neurons was measured $( \pm \mathrm{SEM})$ for at least three separate experiments. For neurons grown on myelin, results are presented as percentage of $\mathrm{P} 1$ neurite length in the absence of cyclic nucleotides $(B)$. For neurons grown on cells, results are presented as percentage of neurite length on control cells in the absence of cyclic nucleotides $(C)$.
B

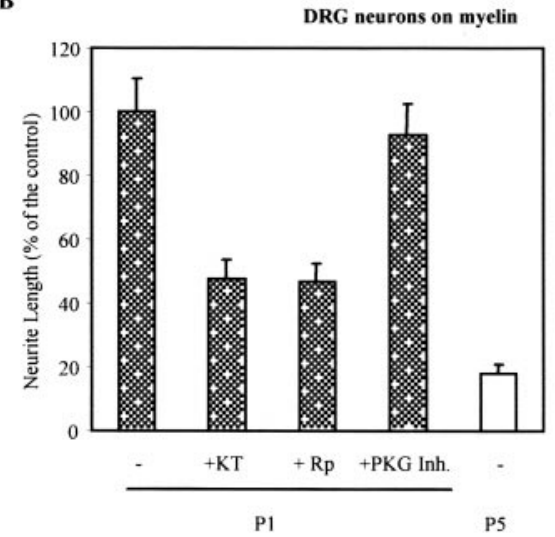

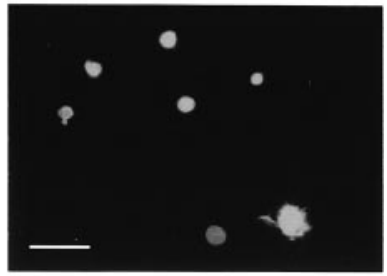

P5

C

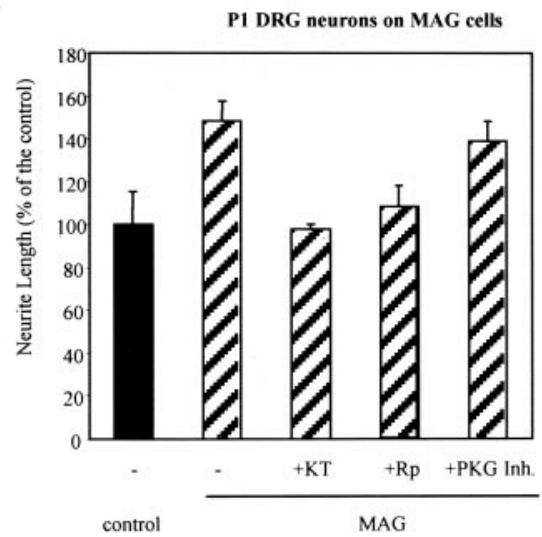

levels of RG neurons are initially high in embryonic neurons and drop to low levels by birth, consistent with the developmental change measured for the RG cell population as a whole. Therefore, like DRG neurons, both the promotion and the inhibition of regeneration by myelin and MAG of RG neurons is cAMP dependent, and the developmental switch in response to myelinMAG is dictated by the endogenous levels of cAMP in the neuron.

\section{Regeneration of neonatal spinal tract axons in vivo and in culture is cAMP dependent}

After spinal cord injury in neonatal animals, there is considerable anatomical reorganization of immature pathways and recovery of function (Bregman and Goldberger, 1982, 1983). Depending on the species and the precise timing of the injury, axons of the spinal tracts are at different stages of maturity; some have already reached their target, and others are still in the process of elongating throughout the cord. Regeneration of those more mature axons that have been severed, aberrant rerouting of latedeveloping axons, and sprouting from undamaged axons all contribute to the recovery (Bregman et al., 1989, 1993; BernsteinGoral and Bregman, 1993). In the rat at P1, corticospinal tract axons are still elongating through the spinal cord, whereas for raphespinal axons, most have reached their targets at all spinal cord levels but continue to increase their projections within the gray matter throughout the next several weeks (Bregman, 1987a,b; Bregman et al., 1989). Furthermore, at this time in the rat spinal cord, depending on the level and the particular tract, myelination has begun, and, of the putative myelin inhibitors, at least MAG is expressed, because it is expressed at the initial stages of myelination (Quarles, 1983). There is a critical period for developmental plasticity; the lesion-induced growth seen at P1 is dramatically decreased by P5 and abolished by the end of the first week of age (Bregman et al., 1989). Consistent with this developmental loss in ability to regenerate in vivo, when raphe nucleus neurons are grown in culture, at P1 they are not inhibited by myelin and MAG, but their ability to grow is inhibited by the PKA inhibitor, H89 (Fig. $5 A, C$ ). In contrast, at P5, raphe neurons are inhibited from extending processes on both myelin and MAG, and inhibition is blocked by elevating cAMP (Fig. $5 B, C)$. As for DRG and $\mathrm{RG}$ neurons, the switch of raphe neurons to inhibition by myelin-MAG is paralleled by a significant drop in endogenous cAMP levels of about threefold between P0 and P5 (Fig. 5D). Therefore, like DRG and RG axons, raphespinal axons switch their response to myelin-MAG during development, and this switch is closely matched with a decrease in their endogenous cAMP levels.

Next, we tested whether spontaneous regeneration and growth of neonatal rat spinal cord is cAMP dependent. P2-3 rat spinal cords were lesioned by overhemisection (all axons in one half of the spinal cord are lesioned along with all dorsal column axons), and the PKA inhibitor, H89, was applied. We have shown previously that implantation of embryonic spinal cord tissue into the lesion site enhances both survival and regeneration at P3 and P8 but does not alter the general outcome; at P3, spinal axons grow into and through the implanted tissue, whereas at P8 axons grow only a short distance into the implanted tissue and never out into the distant host tissue (Bregman, 1987a; Bregman et al., 1989; Bernstein-Goral and Bregman, 1993). Therefore, the H89 was applied either directly to the lesion site via Gelfoam or to the embryonic grafted tissue, also via Gelfoam. After 2 weeks, corticospinal axons were labeled by anterograde tracing methods, and raphespinal tract axons were labeled by immunostaining for serotonin. The treatment with H89 did not alter the survival of the transplants or their apposition with the host tissue. Also, the morphology, as assessed by staining with cresyl violet, of the transplanted cells was unchanged by H89 treatment (data not shown). Healthy transplants with areas of good apposition in the host spinal cord were identified in 5 of 7 lesion plus transplant 
$\mathbf{A}$

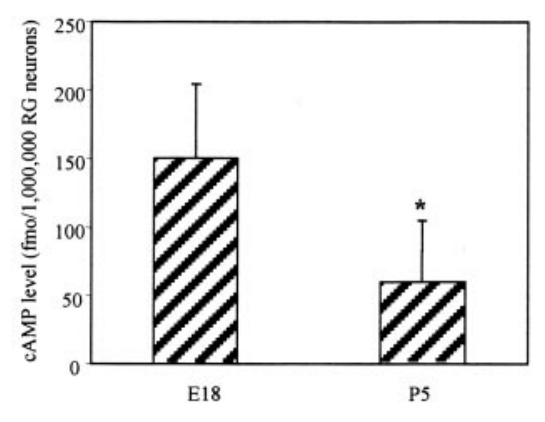

C

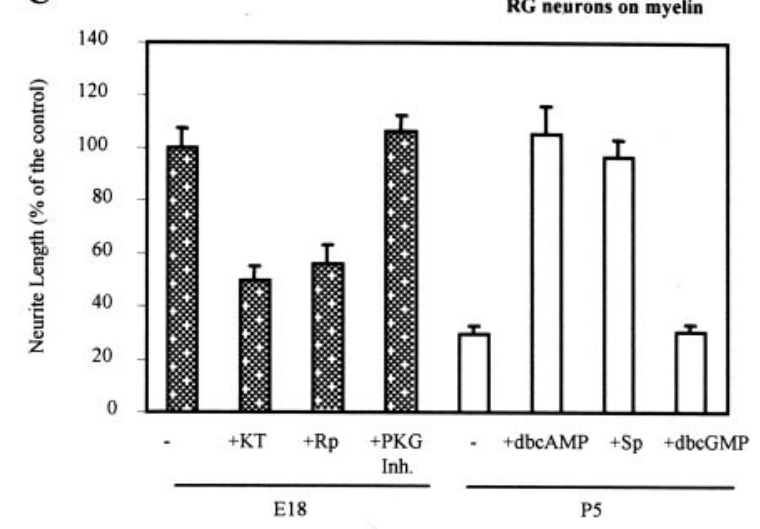

B
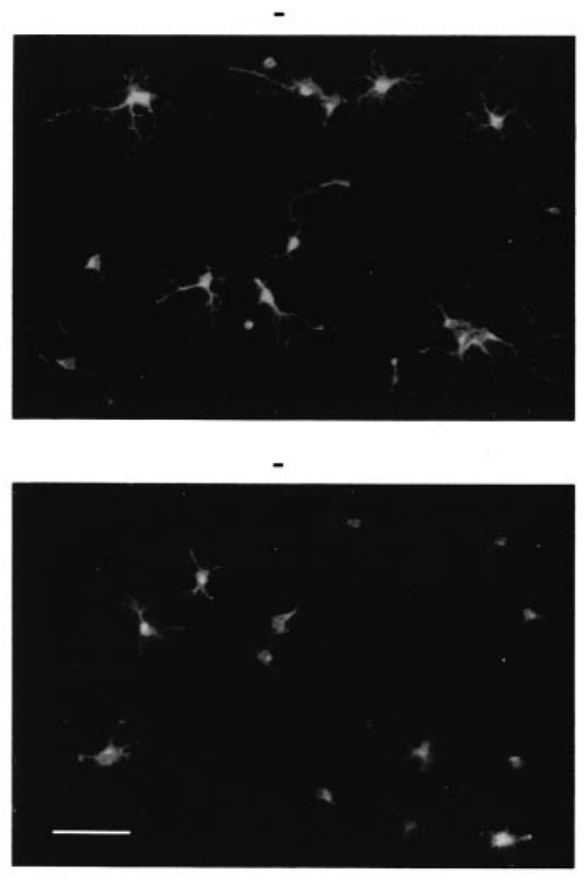

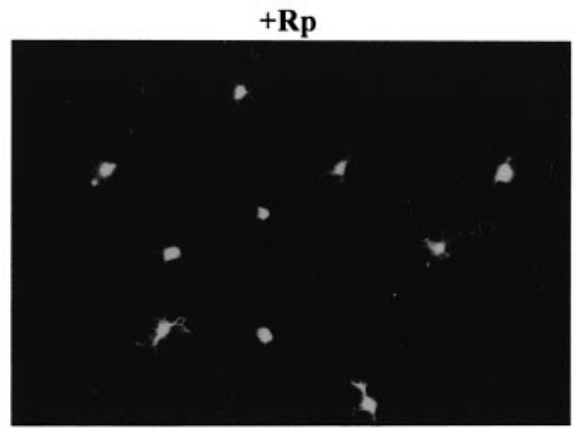

+dbcAMP

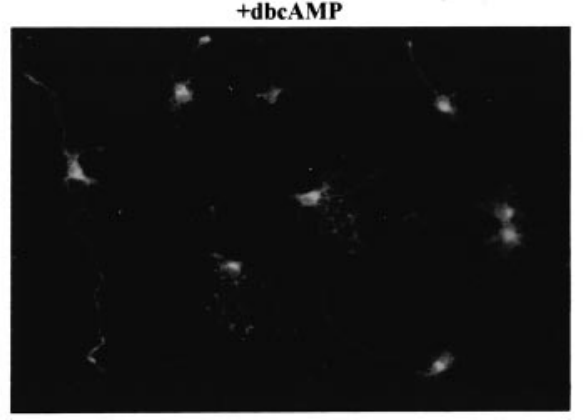

Figure 3. Developmental changes in neurite outgrowth of RG neurons on myelin correlate with, and are dependent on, changes in endogenous levels of cAMP. Dissociated RG cells from E18 or P5 animals were measured for cAMP $\left(1 \times 10^{6}\right.$ cells per well $)$ using a competitive immunoassay. Results are the mean $( \pm \mathrm{SE})$ of at least three experiments, each performed in sextuplicate $(A)$. E18 or P5 RG neurons were cultured overnight on a substrate of purified myelin $(B, C)$. Where indicated, $200 \mathrm{~nm}$ KT5720 $(+K T), 20 \mu \mathrm{M}$ Rp-cAMP $(+R p), 1$ $\mu \mathrm{M} \mathrm{KT} 5823$ (+PKG Inh. $), 1 \mathrm{mM}$ dbcAMP, $50 \mu \mathrm{M}$ Sp-cAMP, or $1 \mathrm{~mm}$ db-cGMP was added during culture as described in Figure 2, after which the neurons were fixed and immunostained for GAP43. Scale bar, $10 \mu \mathrm{m}$. Neurite length was measured for 180 200 neurons $( \pm$ SEM) in each experiment, and results are from at least three experiments. Results are presented as percentage of E18 neurite length, in the absence of cyclic nucleotides. C, Stippled bars represent E18 neurons; white bars represent P5 neurons. ${ }^{*} p<0.05$. animals treated with saline $(71 \%)$ and in 8 of 11 lesion plus transplant animals treated with $\mathrm{H} 89$ (73\%). This rate of survival was equivalent to that observed in earlier studies from this laboratory and was similar in H89- and saline-treated groups. The axonal growth in the H89-treated animals was compared with animals treated with saline. In most of the animals treated with H89 $(n=21)$, a visual difference from control $(n=17)$ was immediately apparent, in both the length and the number of corticospinal and raphespinal axons at the lesion or lesion plus transplant site. Results from a typical experiment for corticospinal axons are shown in Figure 6. In the H89-treated animals, the axonal growth characteristic of developmental plasticity was greatly attenuated in both the lesion only and lesion plus transplant animals. The dense axonal growth characteristic of early lesions was decreased markedly in 18 of the 21 H89-treated animals (Fig. 6A--D; arrows in $D$ point to the little regeneration that is apparent in the H89-treated animals). Similar results were obtained for the raphespinal tract axons that were stained for serotonin (data not shown). The extent of axonal growth within the transplants was scored for the saline- and H89-treated groups (Table 1). These results clearly show that the spontaneous regen- eration and continued growth of young spinal cord axons in vivo is cAMP dependent.

\section{DISCUSSION}

This study represents the first step toward a molecular understanding of the switch during development that results in the inhibition of axonal regeneration by myelin and consequently the inability to regenerate in vivo. Here, we show that the endogenous level of cAMP in three different types of neuron drops dramatically with development and that this spontaneous decrease in cAMP coincides with a switch to the inhibition of regeneration by myelin-MAG. We also show that inhibition in older neurons can be prevented by elevating cAMP and that inhibiting a signal downstream of cAMP activation (inhibition of PKA) can block early developmental promotion of axonal regeneration by myelin-MAG. Consistent with their relatively high levels of cAMP and ability to grow well on myelin and MAG in culture, the ability of young axons to spontaneously regenerate after injury in vivo is also dependent on activation of PKA. These results are important for two reasons. First, they identify for the first time a molecular difference between young and adult neurons that determines their 

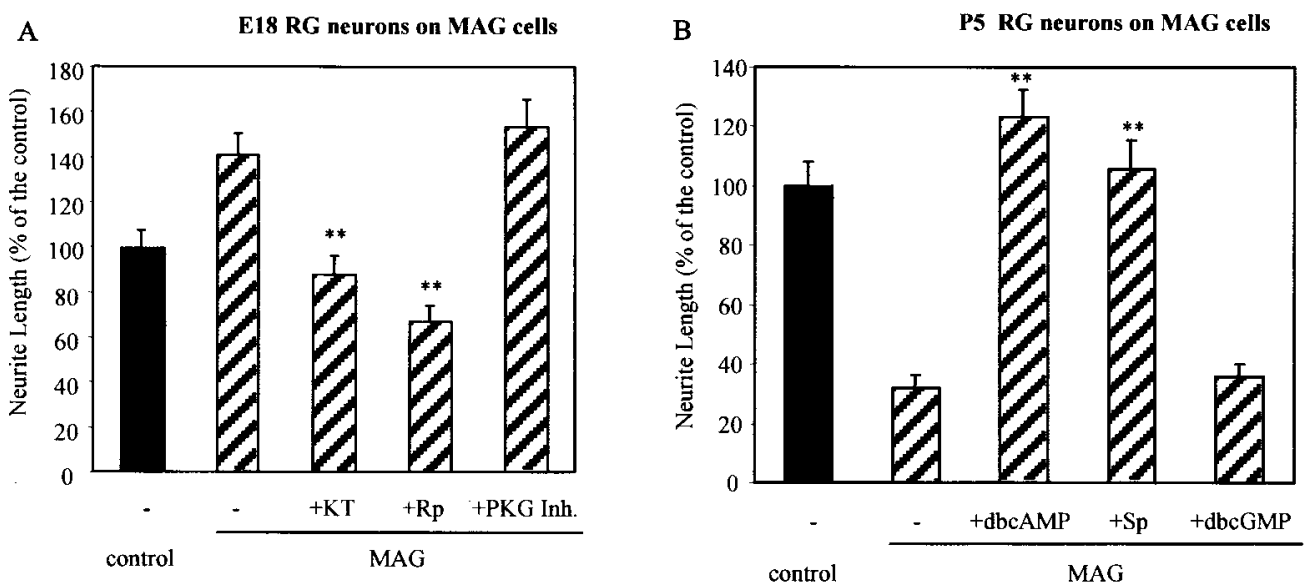

Figure 4. Developmental changes in neurite outgrowth of RG neurons on MAG are dependent on changes in endogenous levels of cAMP. Dissociated RG neurons from E18 $(A)$ or P5 $(B)$ animals were cultured overnight on a substrate of MAG-expressing cells (striped bars) or control cells $($ black bars). Where indicated, $200 \mathrm{~nm}$ KT5720 $(+K T), 20 \mu \mathrm{M}$ Rp-cAMP $(+R p), 1 \mu \mathrm{M} \mathrm{KT5823}(+P K G$ Inh.), 1 mM db-cAMP, $50 \mu \mathrm{M}$ Sp-cAMP $(+S p)$, or 1 mM $\mathrm{db}-\mathrm{cGMP}$ was added during culture; afterward, they were fixed and immunostained for GAP43. In each experiment, the mean length of the longest GAP43-positive neurite for 180-200 neurons was measured ( \pm SEM) for at least three separate experiments. Results are presented as a percentage of neurite length on control cells in the absence of cyclic nucleotides. ${ }^{* *} p<0.001$.

Figure 5. Developmental changes in neurite outgrowth of raphespinal neurons on MAG and myelin are dependent on changes in endogenous levels of cAMP. Raphe nucleus neurons were dissociated from $\mathrm{P} 0-\mathrm{P} 1$ and $\mathrm{P} 4-\mathrm{P} 8$ rat pups and grown on either MAGexpressing (striped bars) or control CHO cells (black bars) $(A, B)$ or purified myelin $(C)$. Where indicated, H89 $(2 \mu \mathrm{M}), \mathrm{KT} 5720(200 \mathrm{nM})$, db-cAMP (1 $\mathrm{mM})$, Sp-cAMP $(50 \mu \mathrm{M})$, or inhibitorsagonists of cGMP were added. Results represent the average neurite length of 180-200 GAP43-positive neurites per experiment from three separate experiments. For neurons grown on myelin, results are presented as percentage of $\mathrm{P} 0-\mathrm{P} 1$ neurite length, and for neurons grown on cells, results are presented as a percentage of neurite length on control cells, each in the absence of cyclic nucleotides. $D$, Dissociated raphe cells from $\mathrm{P} 0-\mathrm{P} 1$ or P3-8 animals were measured for cAMP $\left(2 \times 10^{5}\right.$ cells per well $)$ using a competitive immunoassay. Results are the mean $( \pm \mathrm{SE})$ of at least three experiments, each performed in sextuplicate. ${ }^{*} p<0.05 ;{ }^{* *} p<0.001$.

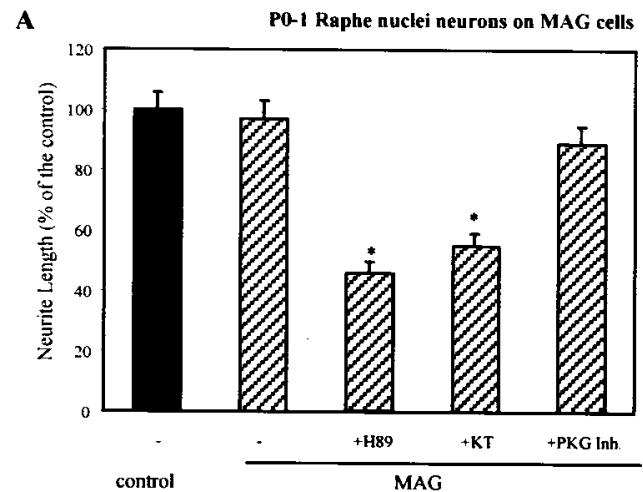

B

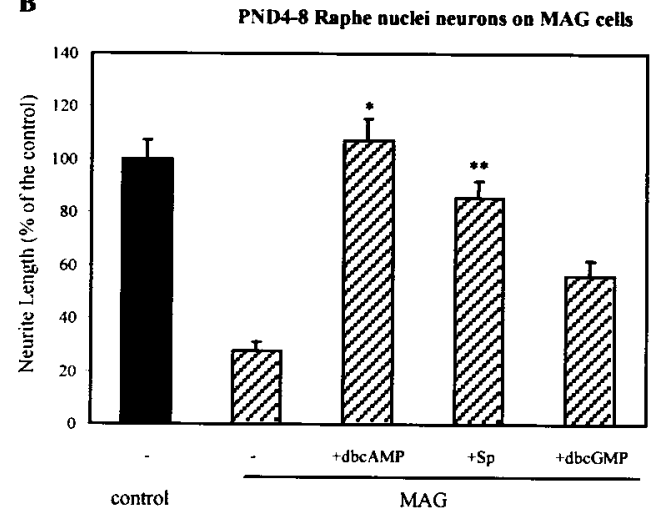

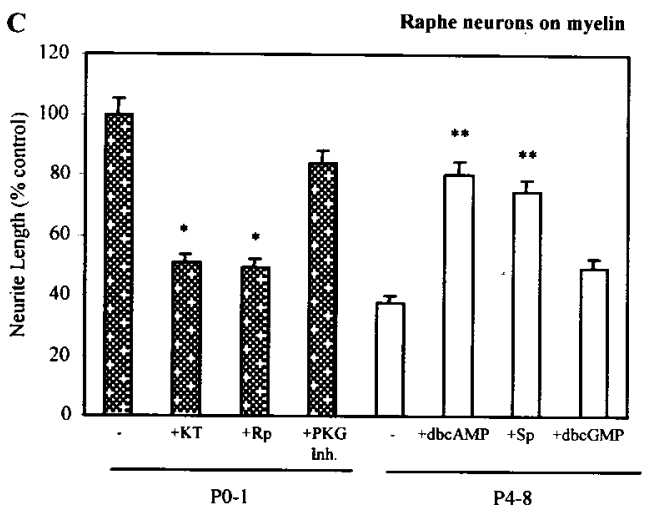

D

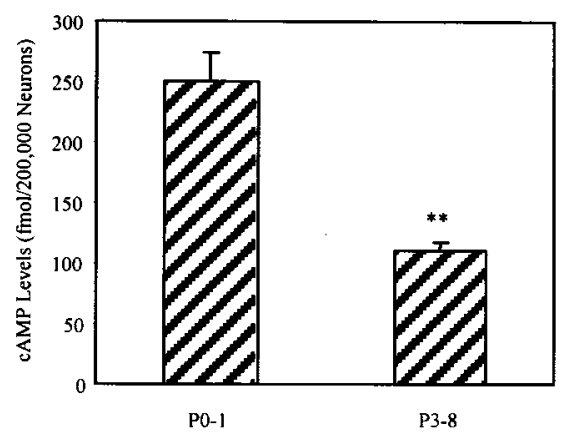

response to inhibitors in myelin and consequently their ability to regenerate. The strong implication is that artificially adjusting cAMP levels or manipulating PKA activity in adult neurons may offer a means to support regeneration after injury. Second, the results demonstrate that the physiological levels of cAMP change sufficiently with development to the point at which they can effect the switch from promotion to inhibition in response to myelinMAG. Others have shown in vitro that altering cAMP levels can reverse the turning of Xenopus growth cones in response to a number of different guidance cues (Ming et al., 1997; Song et al.,
1997, 1998). The relevance of those observations to events in vivo was not demonstrated previously. In addition, neither was it shown that the changes in cAMP necessary for such dramatic switches in guidance response were inside the physiological range in vivo. Here, we show, at least for myelin and MAG, that these changes are well within physiological ranges. Therefore, it is highly likely that endogenous cAMP levels determine the guidance response to other molecules in vivo.

It should be noted that a second group of guidance cues, although independent of cAMP, are dependent on the cyclic 

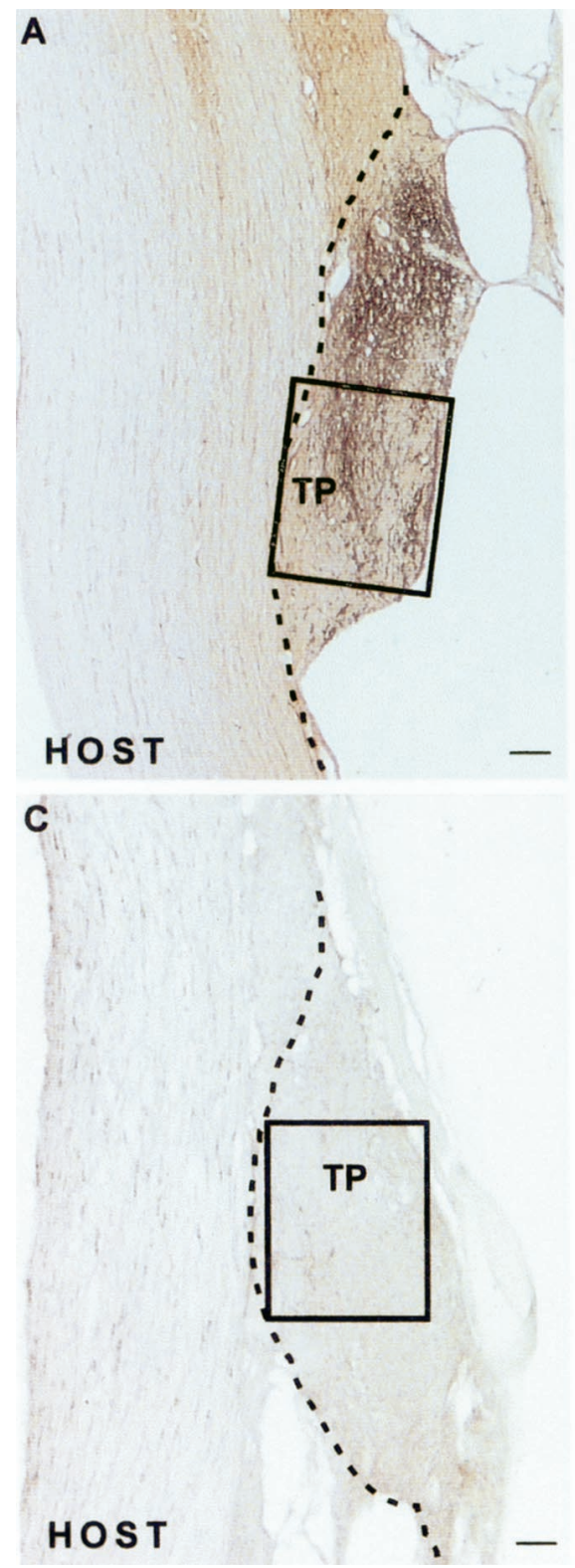
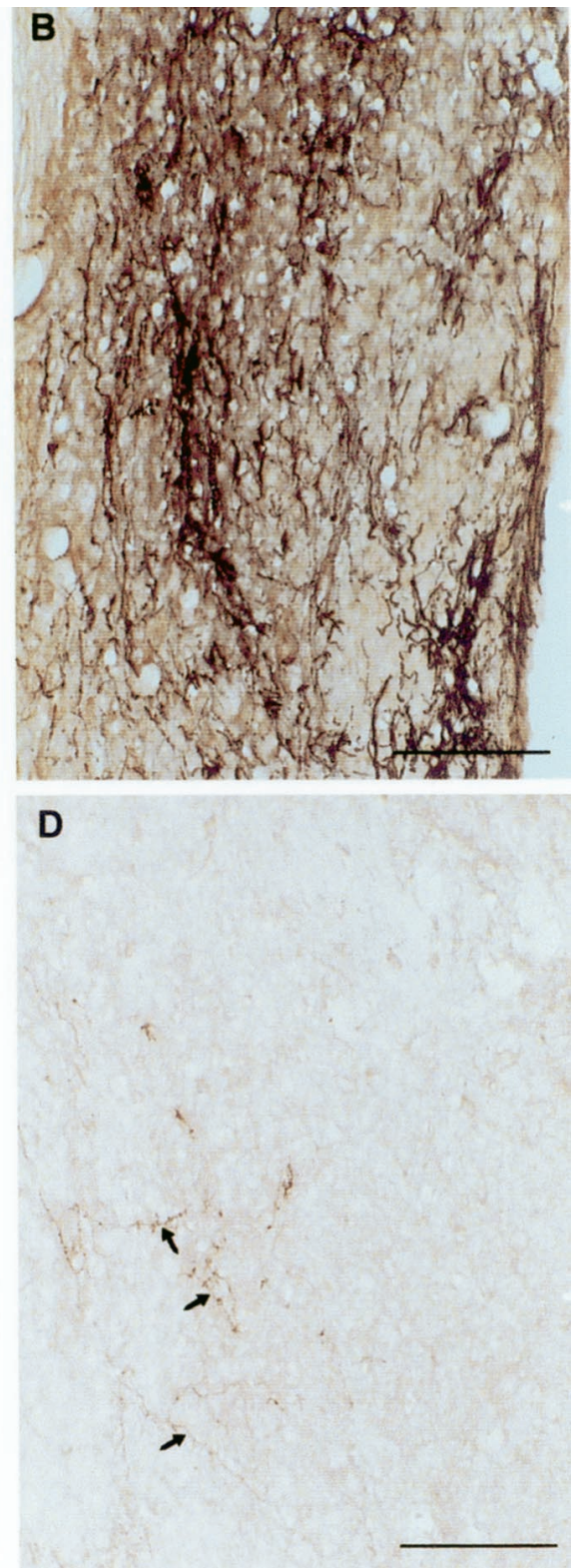

Figure 6. Regeneration of neonatal spinal axons in vivo is cAMP-dependent. $\mathrm{P} 2-3$ rat pups were subjected to overhemisection lesion of their spinal cords at T6. Embryonic spinal cord transplants were placed into the lesion site without $(A, B)$ or with H89 $(0.5$ $\mathrm{mm})(C, D)$. Corticospinal axons were labeled anterogradely with BDA, and animals were killed at 4 weeks after transplantation. Dashed lines $(A, C)$ indicate the approximate border between host and transplant $(T P)$ tissue, and the boxed areas in $A$ and $C$ are shown at higher magnification in $B$ and $D$, respectively. Corticospinal axon growth within the transplant is dramatically decreased in the presence of H89. Arrows in $C$ indicate the few axons that regenerated into the TP in the H89-treated animal. A similar decrease in axonal growth by $\mathrm{H} 89$ was also seen for serotonergic axons (data not shown). Scale bars, $100 \mu \mathrm{m}$.
Table 1. Scoring axonal growth within transplant

\begin{tabular}{llll}
$\begin{array}{c}\text { Animal } \\
\text { number }\end{array}$ & $\mathrm{HX}+\mathrm{TP}+$ saline & $\begin{array}{l}\text { Animal } \\
\text { number }\end{array}$ & $\mathrm{HX}+\mathrm{TP}+\mathrm{H} 89$ \\
\hline 1 & ++ & 1 & 0 \\
2 & +++ & 2 & 0 \\
3 & +++ & 3 & + \\
4 & +++ & 4 & + \\
5 & +++ & 5 & + \\
& & 6 & ++ \\
& & 7 & ++
\end{tabular}

Transection and transplantation with and without H89 are as described in Fig. 6. HX, Overhemisection; TP, transplant. Rating scale: $0=$ no axon growth; $+=$ scattered fibers in transplant near host-transplant border; $++=$ sparse fibers throughout transplant; $+++=$ dense fibers throughout transplant. nucleotide cGMP for dictating the response of the neuron (Ming et al., 1997). Importantly, Polleux et al. (2000) showed recently that axons of pyramidal neurons are repelled by the guidance molecule, Sem3A, whereas dendrites of the same neuron are attracted by Sem3A. Asymmetric distribution of the cGMP synthesizing enzyme, guanylate cyclase, and hence, presumably, of cGMP, is responsible for the opposite effects of Sema3A on axons and dendrites; cGMP is high in dendrites and low in the axon. Our findings here indicate that inhibition not only by MAG but also by myelin inhibitors in general, which must include the recently described inhibitor Nogo (Chen et al., 2000; GrandPre et al., 2000), are largely cAMP dependent. Recently, it was shown that inactivation of the small GTPase, Rho, not only blocked inhibition by MAG and myelin but also allowed regeneration in vivo (Lehmann et al., 1999). It is possible that cAMP-dependent activation of PKA has a direct effect on Rho signaling. In addition, because all neuronal types tested to date respond by either inhibition or promotion, depending on age, we can conclude that they all express the myelin-MAG receptor(s). Because cAMP 
levels are sufficient to determine whether inhibition or promotion of neurite outgrowth occurs, the myelin-MAG neuronal receptor(s) is likely to be identical for each effect and in each type of neuron. For other guidance cues that are reversed by altering cAMP signaling, the importance of the cytoplasmic domains of certain receptors in determining the response to those cues has been demonstrated (Bashaw and Goodman, 1999; Hong et al., 1999). However, it is possible that signaling through particular receptors directly alters cyclic nucleotide levels and so influences whether there is an attractive or repulsive effect. Indeed, a coreceptor for one such bifunctional guidance cue, Netrin, is the A2b adenosine receptor that, when activated, directly affects cellular cAMP levels (Corset et al., 2000).

In addition to inhibitors in myelin, other factors also affect regeneration in vivo (Schwab and Bartholdi, 1996). The formation of the glial scar is a major obstacle to regrowth. However, the glial scar takes time to form, and the recent report of regeneration in myelin-immunized mice clearly shows that myelin components stop growth immediately after injury; their neutralization with antibodies allows growth before the scar forms (Huang et al., 1999). In addition, adult neurons have been shown to extend long axons when they are transplanted into the adult CNS with such care that no scar forms (Davies et al., 1997, 1999). Importantly, under these conditions, myelin is not damaged. Consequently, these axons grow on the outer surface of undamaged myelin, which contains molecules shown to be permissive for growth (Turnley and Bartlett, 1998). Myelin inhibitors, therefore, are only exposed after damage.

What causes the decline in the endogenous levels of cAMP in neurons with development? There could be some developmental switch in enzymes that synthesize or degrade cAMP. Isoforms of PKA may change with development. This could affect the "free" levels of cAMP and the threshold level of cAMP required for PKA activation. Alternatively, we showed previously that the inhibition of axonal regeneration by myelin-MAG could be blocked if neurons were exposed to various neurotrophins before their exposure to the inhibitor, a process we termed "priming with neurotrophin" (Cai et al., 1999). Furthermore, the block of inhibition by priming with neurotrophin was cAMP dependent, and the neurotrophins used were shown to elevate neuronal cAMP levels. Coupled with the demonstration that expression in vivo of both neurotrophins and their receptors (along with peptides and hormones more traditionally regarded as cAMP activators) declines with development (Jelsma and Aguayo, 1994), these observations raise the likelihood that neurotrophins and growth factors in the environment regulate the cAMP levels of neurons. The same molecules can then be seen to dictate the response to myelin-MAG. Because there is strong evidence implicating cAMP-dependent events in plasticity (Bailey et al., 1996), it is notable that the switch in response of neurons to myelin-MAG accompanies, at about essentially the same time, the developmental loss of neuronal plasticity. One possibility is that the spontaneous decrease in neuronal cAMP not only mediates the switch from promotion to inhibition of axonal regeneration by myelinMAG but also leads to the loss of neuronal plasticity. Interestingly, the physiological function of inhibition by myelin-MAG is suggested to control sprouting (Schwegler et al., 1995), a phenomenon closely associated with neuronal plasticity. Endogenous cAMP levels may also affect neuronal survival because elevating cAMP has been shown to potentiate the effects of a number of neurotrophins (Meyer-Franke et al., 1995; Hanson et al., 1998; Shen et al., 1999). However, the ability of elevated cAMP to overcome inhibition by myelin-MAG is not purely a consequence of improved survival because elevated cAMP does not affect axonal growth on control cells. In addition, for RG neurons at least, elevated cAMP alone is insufficient to improve survival (Shen et al., 1999). It is also of note that mice overexpressing the anti-apoptotic protein, bcl-2, show improved survival but no improved regenerative capacity (Chierzi et al., 1999). Finally, all of these cAMP-affected phenomena (regeneration, plasticity, and survival) are connected in that they switch with development, are likely to share similar mechanisms, and are all required for functional recovery after injury.

\section{REFERENCES}

Bailey CH, Bartsch D, Kandel ER (1996) Toward a molecular definition of long-term memory storage. Proc Natl Acad Sci USA 93:13445-13452.

Bashaw GJ, Goodman CS (1999) Chimeric axon guidance receptors: the cytoplasmic domains of slit and netrin receptors specify attraction versus repulsion. Cell 97:917-926.

Bates CA, Stelzner DJ (1993) Extension and regeneration of corticospinal axons after early spinal injury and the maintenance of corticospinal topography. Exp Neurol 123:106-117.

Bernstein-Goral H, Bregman BS (1993) Spinal cord transplants support the regeneration of axotomized neurons after spinal cord lesions at birth: a quantitative double-labeling study. Exp Neurol 123:118-132.

Bregman BS (1987a) Spinal cord transplants permit the growth of serotonergic axons across the site of neonatal spinal cord transection. Brain Res 431:265-279.

Bregman BS (1987b) Development of serotonin immunoreactivity in the rat spinal cord and its plasticity after neonatal spinal cord lesions. Brain Res 431:245-263.

Bregman BS, Goldberger ME (1982) Anatomical plasticity and sparing of function after spinal cord damage in neonatal cats. Science 217:553-555.

Bregman BS, Goldberger ME (1983) Infant lesion effect: III. Anatomical correlates of sparing and recovery of function after spinal cord damage in newborn and adult cats. Brain Res 285:137-154.

Bregman BS, McAtee M (1993) Embryonic CNS tissue transplantation for studies of development and regeneration. Neuroprotocols 3:17-27.

Bregman BS, Kunkel-Bagden E, McAtee M, O’Neill A (1989) Extension of the critical period for developmental plasticity of the corticospinal pathway. J Comp Neurol 282:355-370.

Bregman BS, Kunkel-Bagden E, Reier PJ, Dai HN, McAtee M, Gao D (1993) Recovery of function after spinal cord injury: mechanisms underlying transplant-mediated recovery of function differ after spinal cord injury in newborn and adult rats. Exp Neurol 123:3-16.

Bregman BS, McAtee M, Dai HN, Kuhn PL (1997) Neurotrophic factors increase axonal growth after spinal cord injury and transplantation in the adult rat. Exp Neurol 148:475-494.

Cai D, Shen Y, De Bellard M, Tang S, Filbin MT (1999) Prior exposure to neurotrophins blocks inhibition of axonal regeneration by MAG and myelin via a cAMP-dependent mechanism. Neuron 22:89-101.

Chen MS, Huber AB, van der Haar ME, Frank M, Schnell L, Spillmann AA, Christ F, Schwab ME (2000) Nogo-A is a myelin-associated neurite outgrowth inhibitor and an antigen for monoclonal antibody IN-1. Nature 403:434-439.

Cheng H, Cao Y, Olson L (1996) Spinal cord repair in adult paraplegic rats: partial restoration of hind limb function. Science 273:510-513.

Chierzi S, Strettoi E, Cenni MC, Maffei L (1999) Optic nerve crush: axonal responses in wild-type and bcl-2 transgenic mice. J Neurosci 19:8367-8376.

Colamarino SA, Tessier-Lavigne M (1995) The axonal chemoattractant netrin-1 is also a chemorepellent for trochlear motor axons. Cell 81:621-629.

Corset V, Nguyen-Ba-Charvet KT, Forcet C, Moyse E, Chedotal A, Mehlen P (2000) Netrin-1-mediated axon outgrowth and cAMP production requires interaction with adenosine $\mathrm{A} 2 \mathrm{~b}$ receptor. Nature 407:747-750.

Crutcher KA (1989) Tissue sections from the mature rat brain and spinal cord as substrates for neurite outgrowth in vitro: extensive growth on gray matter but little growth on white matter. Exp Neurol 104:39-54.

David S, Aguayo AJ (1981) Axonal elongation into peripheral nervous system "bridges" after central nervous system injury in adult rats. Science 214:931-933.

Davies SJ, Fitch MT, Memberg SP, Hall AK, Raisman G, Silver J (1997) Regeneration of adult axons in white matter tracts of the central nervous system. Nature 390:680-683.

Davies SJ, Goucher DR, Doller C, Silver J (1999) Robust regeneration of adult sensory axons in degenerating white matter of the adult rat spinal cord. J Neurosci 19:5810-5822.

DeBellard ME, Tang S, Mukhopadhyay G, Shen YJ, Filbin MT (1996) 
Myelin-associated glycoprotein inhibits axonal regeneration from a variety of neurons via interaction with a sialoglycoprotein. Mol Cell Neurosci 7:89-101.

Diener PS, Bregman BS (1998) Fetal spinal cord transplants support growth of supraspinal and segmental projections after cervical spinal cord hemisection in the neonatal rat. J Neurosci 18:779-793.

GrandPre T, Nakamura F, Vartanian T, Strittmatter SM (2000) Identification of the Nogo inhibitor of axon regeneration as a Reticulon protein. Nature 403:439-444.

Hanson MG Jr, Shen S, Wiemelt AP, McMorris FA, Barres BA (1998) Cyclic AMP elevation is sufficient to promote the survival of spinal motor neurons in vitro. J Neurosci 18:7361-7371.

Hasan SJ, Keirstead HS, Muir GD, Steeves JD (1993) Axonal regeneration contributes to repair of injured brainstem-spinal neurons in embryonic chick. J Neurosci 13:492-507.

Herzog A, Brosamle C (1997) 'Semifree-floating' treatment: a simple and fast method to process consecutive sections for immunohistochemistry and neuronal tracing. J Neurosci Methods 72:57-63.

Hong K, Hinck L, Nishiyama M, Poo MM, Tessier-Lavigne M, Stein E (1999) A ligand-gated association between cytoplasmic domains of UNC5 and DCC family receptors converts netrin-induced growth cone attraction to repulsion. Cell 97:927-941.

Huang DW, McKerracher L, Braun PE, David S (1999) A therapeutic vaccine approach to stimulate axon regeneration in the adult mammalian spinal cord. Neuron 24:639-647.

Jelsma TN, Aguayo AJ (1994) Trophic factors. Curr Opin Neurobiol 4:717-725.

Johnson PW, Abramow-Newerly W, Seilheimer B, Sadoul R, Tropak MB, Arquint M, Dunn RJ, Schachner M, Roder JC (1989) Recombinant myelin-associated glycoprotein confers neural adhesion and neurite outgrowth function. Neuron 3:377-385.

Keirstead HS, Hasan SJ, Muir GD, Steeves JD (1992) Suppression of the onset of myelination extends the permissive period for the functional repair of embryonic spinal cord. Proc Natl Acad Sci USA 89:11664-11668.

Lehmann M, Fournier A, Selles-Navarro I, Dergham P, Sebok A, Leclerc N, Tigyi G, McKerracher L (1999) Inactivation of rho signaling pathway promotes CNS axon regeneration. J Neurosci 19:7537-7547.

Li Y, Raisman G (1993) Long axon growth from embryonic neurons transplanted into myelinated tracts of the adult rat spinal cord. Brain Res 629:115-127.

McKerracher L, David S, Jackson DL, Kottis V, Dunn RJ, Braun PE (1994) Identification of myelin-associated glycoprotein as a major myelin-derived inhibitor of neurite growth. Neuron 13:805-811.

Meyer-Franke A, Kaplan MR, Pfrieger FW, Barres BA (1995) Characterization of the signaling interactions that promote the survival and growth of developing retinal ganglion cells in culture. Neuron 15:805-819.

Ming GL, Song HJ, Berninger B, Holt CE, Tessier-Lavigne M, Poo MM (1997) cAMP-dependent growth cone guidance by netrin-1. Neuron 19:1225-1235.

Mukhopadhyay G, Doherty P, Walsh FS, Crocker PR, Filbin MT (1994) A novel role for myelin-associated glycoprotein as an inhibitor of axonal regeneration. Neuron 13:757-767.

Polleux F, Morrow T, Ghosh A (2000) Semaphorin 3A is a chemoattractant for cortical apical dendrites. Nature 404:567-573.

Quarles RH (1983) Myelin-associated glycoprotein in development and disease. Dev Neurosci 6:285-303.

Richardson PM, McGuinness UM, Aguayo AJ (1980) Axons from CNS neurons regenerate into PNS grafts. Nature 284:264-265.

Salzer JL, Pedraza L, Brown M, Struyk A, Afar D, Bell J (1990) Structure and function of the myelin-associated glycoproteins. Ann N Y Acad Sci 605:302-312.

Schnell L, Schwab ME (1990) Axonal regeneration in the rat spinal cord produced by an antibody against myelin-associated neurite growth inhibitors. Nature 343:269-272.

Schwab ME, Bartholdi D (1996) Degeneration and regeneration of axons in the lesioned spinal cord. Physiol Rev 76:319-370.

Schwab ME, Kapfhammer JP, Bandtlow CE (1993) Inhibitors of neurite growth. Annu Rev Neurosci 16:565-595.

Schwegler G, Schwab ME, Kapfhammer JP (1995) Increased collateral sprouting of primary afferents in the myelin-free spinal cord. J Neurosci $15: 2756-2767$.

Shen S, Wiemelt AP, McMorris FA, Barres BA (1999) Retinal ganglion cells lose trophic responsiveness after axotomy. Neuron 23:285-295.

Shewan D, Berry M, Cohen J (1995) Extensive regeneration in vitro by early embryonic neurons on immature and adult CNS tissue. J Neurosci 15:2057-2062.

Song H, Ming G, He Z, Lehmann M, McKerracher L, Tessier-Lavigne M, Poo M (1998) Conversion of neuronal growth cone responses from repulsion to attraction by cyclic nucleotides. Science 281:1515-1518.

Song HJ, Ming GL, Poo MM (1997) cAMP-induced switching in turning direction of nerve growth cones. Nature [Erratum (1997) 389:412] 388:275-279.

Turnley AM, Bartlett PF (1998) MAG and MOG enhance neurite outgrowth of embryonic mouse spinal cord neurons. NeuroReport 9:1987-1990.

Veenman CL, Reiner A, Honig MG (1992) Biotinylated dextran amine as an anterograde tracer for single- and double-labeling studies. J Neurosci Methods 41:239-254

Wiemelt AP, Engleka MJ, Skorupa AF, McMorris FA (1997) Immunochemical visualization and quantitation of cyclic AMP in single cells. J Biol Chem 272:31489-31495. 